\title{
Assessment of image resolution improvement by phase-sensitive optical parametric amplification
}

\author{
Z. Huang • D. French · H.-Y. Pao • I. Jovanovic
}

Received: 25 April 2010 / Revised version: 25 May 2010 / Published online: 18 June 2010

(C) The Author(s) 2010. This article is published with open access at Springerlink.com

\begin{abstract}
Classical information theory can be used to quantify the resolution performance of optical imaging systems. When an optical parametric amplifier (OPA) operated as a phase-sensitive amplifier (PSA) in the transverse spatial domain is used for point source imaging, the angular resolution improvement can approach the de Broglie resolution (i.e. Heisenberg limit). In this paper, classical information theory is employed to quantify the signal-to-noise ratio (SNR) improvement for both an ideal and a realistic multimode PSA applied to the problem of sub-Rayleigh imaging. When only considering the noise originating from the detector, the SNR improvement is found to scale quadratically as a function of the PSA gain, in the limit of noise power comparable to signal power. Differences in performance of an ideal PSA and a realistic PSA are discussed.
\end{abstract}

\section{Introduction}

The resolution of an imaging system is usually represented by the ability to distinguish between two points exhibiting a small angular separation. The Rayleigh criterion specifies the minimum angular separation that can be resolved, and represents one of the usual metrics of resolution. According to the Rayleigh criterion, the minimum resolvable angular separation, $\theta_{\mathrm{R}}$, is given by $\theta_{\mathrm{R}}=1.22 \lambda / D$, where $\lambda$ is the wavelength of the optical signal, and $D$ is the circular

Z. Huang · D. French · I. Jovanovic $(\varangle)$

School of Nuclear Engineering, Purdue University,

West Lafayette, IN 47907, USA

e-mail: ijovanovic@purdue.edu

H.-Y. Pao

Lawrence Livermore National Laboratory, PO Box 808,

Livermore, CA 94551, USA aperture diameter of the imaging system. The Rayleigh criterion represents an arbitrary measure of resolution; a more rigorous approach to quantifying the resolution of an imaging system is the use of the signal-to-noise ratio (SNR). For two point sources separated by a distance $2|u|$, and with the power of each point source of $P_{\mathrm{s}}$, the threshold SNR has been previously shown to be [1]:

$\mathrm{SNR} \propto|u|^{4} P_{\mathrm{s}}^{2}$.

If $\theta \propto|u|$ is the small departure angle of each point source from the normal to the aperture (detector) surface, the minimum departure angle, $\theta_{\min }$, which can be resolved for a fixed SNR scales as $\theta_{\min } \propto 1 / \sqrt{P_{\mathrm{S}}}$, corresponding to the classical shot-noise limit. Significant amount of prior work has been done in the area of quantum imaging [2] with a goal to enhance the resolution beyond the limits achieved by classical sensors by noting that the noiseless image amplification can be achieved in a phase-sensitive amplifier (PSA) [3, 4]. This noiseless amplification has also been experimentally demonstrated using parametric amplifiers [5-7]. One method that has been suggested to improve resolution beyond that achievable by passive optical imaging systems is the use of PSA operated in a phase amplification mode. This mode of operation can be achieved via nonlinear three-wave mixing in an optical parametric amplifier (OPA) configured as a photon number deamplifier [8, 9]. When an ideal, constant deamplification phase relationship exists among the three waves involved in the difference-frequency mixing process, the relative phase of the three waves is preserved. If a phase shift is present in the signal beam such that the signal phase departs from this ideal phase condition, this signal phase shift can be amplified [9]. In our previous work, we have derived the criteria for which a realistic OPA operating on spatially multimode beams with a transversely variable phase shift can be made phase-sensitive [9]. 
Analysis showed that an OPA operated as a PSA can significantly redistribute the spatial frequencies of optical beams in a fashion that allows the discrimination between on-axis and off-axis beams. In other work, a full quantum treatment has been invoked to suggest that phase amplification can be used to reduce the bit error rate (BER) in communication protocols [8]. A quantum mechanical analysis of the squeezed state generated by phase amplification [10] indicates that an improvement of SNR in the temporal domain is expected, when a coherent input state and homodyne receiver are used. Here we apply a formal methodology to assess the impact of the use of the nonlinear transformation of a beam by PSA on the performance of an imaging system. The formal methodology adopted here is the rigorous use of the classical information theory to assess the suggestion that the PSA may also be used to improve spatial resolution in imaging problems by increasing the apparent incident beam angle. Both an ideal PSA and the real PSA realized by an optical parametric amplifier is included in this analysis. The mechanism by which the incident beam angle can be increased by the of PSA is illustrated in Fig. 1, and amounts to the amplification of the phase difference between the incident signal and the reference beam normal to the amplifier aperture. This phase amplification is spatially variable in the direction transverse to beam propagation. A simple single-mode treatment assumption [11] suggests that the SNR in an ideal PSA scales as

$\mathrm{SNR} \propto G^{2}|u|^{4} P_{\mathrm{s}}^{2}=|u|^{4}\left(G P_{\mathrm{s}}\right)^{2}$,

where $G$ is the power deamplification gain of the PSA. Since phase amplification is limited by the ability to deamplify photon number, the maximum gain that can be achieved is $G=P_{\mathrm{s}}$, where $P_{\mathrm{S}}$ is in the units of photon number. In this case, an ideal PSA operated at maximum gain decreases the minimum signal power required to resolve two point sources by $P_{\mathrm{s}}$. Therefore, the minimum angular separation between two point sources, or the angular resolution that can be resolved for a fixed SNR, scales as $1 / P_{\mathrm{s}}$. This shows the PSA has a potential to beat the classical Rayleigh limit and achieve the de Broglie resolution, or Heisenberg limit [12]. We apply our previously developed numerical model for multimode PSA [9] to study the distinguishability problem posed in [1]. The distinguishability problem studied is shown in Fig. 2, and it consists of discriminating between two points, where one of the points is located on-axis, and the other is located off-axis of the imaging system. For the first time to our knowledge, we demonstrate that a realistic, spatially multimode PSA based on phase-sensitive optical parametric amplification can improve distinguishability in imaging systems.
Fig. 1 Principle of transverse angular amplification by PSA. The angles have been exaggerated for clarity. The PSA increases the apparent signal incident angle as measured in reference to the signal angle of the pump
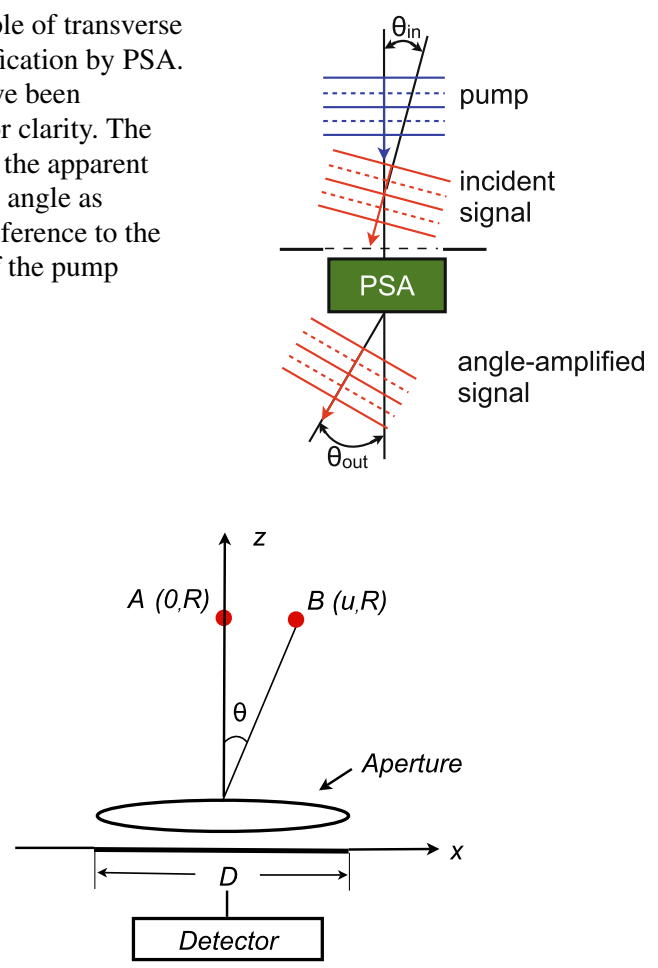

Fig. 2 Schematic of two asymmetrically separated point sources, $A$ and $B$, which are located at $(0, R)$ and $(-u, R)$ with a separation angle $\theta . R$ is the distance from the point source to the center of the aperture with diameter $D$, and the $x$ axis is parallel to the surface of the detector system

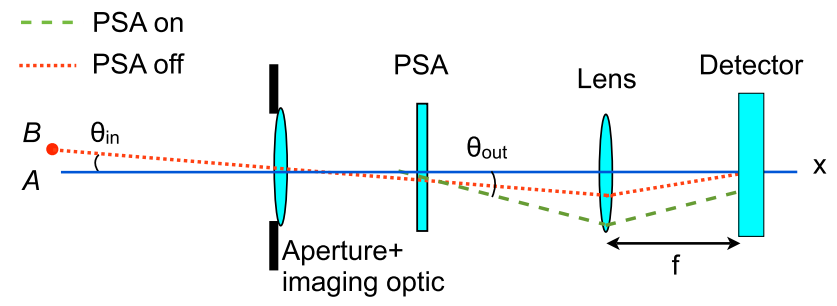

Fig. 3 Schematic of an imaging system that uses a PSA. The incident angular separation of the two point sources to be imaged, $A$ and $B$, is $\theta_{\text {in }}$. With the PSA turned on, the apparent angle between the two point sources increases to $\theta_{\text {out }}$

\section{Application of the phase-sensitive amplification model to the distinguishability problem}

We study a realistic three-wave mixing OPA realized in $\beta$-barium borate (BBO) with $\lambda_{1}=\lambda_{2}=800 \mathrm{~nm}$, and $\lambda_{3}=$ $400 \mathrm{~nm}$, where $\lambda_{1}, \lambda_{2}$, and $\lambda_{3}$ are the wavelengths of the signal, idler, and pump beams, respectively. The incident pump intensity is set as $10 \mathrm{GW} / \mathrm{cm}^{2}$ and the signal/idler intensity is $0.5 \mathrm{GW} / \mathrm{cm}^{2}$. The gain of the OPA is adjusted by varying the crystal thickness. For simplicity, the canonical imaging system used in this study utilizes a one-dimensional aperture, as shown in Fig. 3. The apparent angular separation of the two point sources is increased as a result of the re- 
distribution of spatial frequencies produced by the PSA [9]. This redistribution occurs without increasing in the width of the spatial frequency distribution. This is in stark contrast to the use of a simple magnifying telescope, which would increase the apparent angular separation of two point sources, but also increase the width of the point-spread function associated with the aperture. Thus the increase in angular separation due to PSA is equivalent to conventional imaging of two point sources separated by an angle greater than their original separation angle. For our analysis we choose a typical sub-Rayleigh imaging problem. The effect of PSA on the spatial frequency distributions measured at the detector is calculated and shown in Fig. 4. The distribution in Fig. 4a corresponds to a single point source located at the zenith of the imaging system, or on-axis. The distribution in Fig. 4b corresponds to two point sources, one located on-axis and the other off-axis, with small angular separation compared to the Rayleigh limit, $\theta=\theta_{\mathrm{R}} / 100$. It is evident that for the case in Fig. 4a, the dominant effect of the PSA is to decrease the signal intensity, while for the case in Fig. 4b, the PSA also increases the apparent angular separation of the two points.

\section{Resolution improvement from classical information theory}

Helstrom's classical test is applied to calculate the SNR associated with the distinguishability problem posed in Fig. 2. Two hypotheses can be constructed, corresponding to cases shown in Fig. 4. One hypothesis, $H_{0}$, represents a single point source, Fig. $4 \mathrm{a}$, and the other hypothesis, $H_{1}$, represents two point sources, Fig. 4b. The resolution of two point sources can be determined by a choice between an illuminance $J_{0}$ and $J_{1}$, where $J_{0}$ represents the illuminance due to one point source and its background and $J_{1}$ corresponds to two point sources and their background, respectively [1].

It is assumed that the number of incident photons detected by an optical receiver is shot-noise limited, and thus exhibits a Poisson distribution. The detection is conducted in a one-dimensional geometry with unit quantum efficiency, and the mean number of observed photons at the transverse coordinate $x$ due to hypothesis $H_{k}(k=0,1)$ per unit area and unit time is given by [13]

$\bar{n}_{k}=\int J_{k}(x, \omega) d \omega / \hbar \omega=M_{k}(x)$,

where $J_{k}(x, \omega)$ is the illuminance at angular frequency $\omega$. $M_{k}(x)$ represents the count-rate density at point $x$ and the background illuminance $J_{\mathrm{b}}$ is assumed to be uniform for both $J_{1}$ and $J_{0}$.
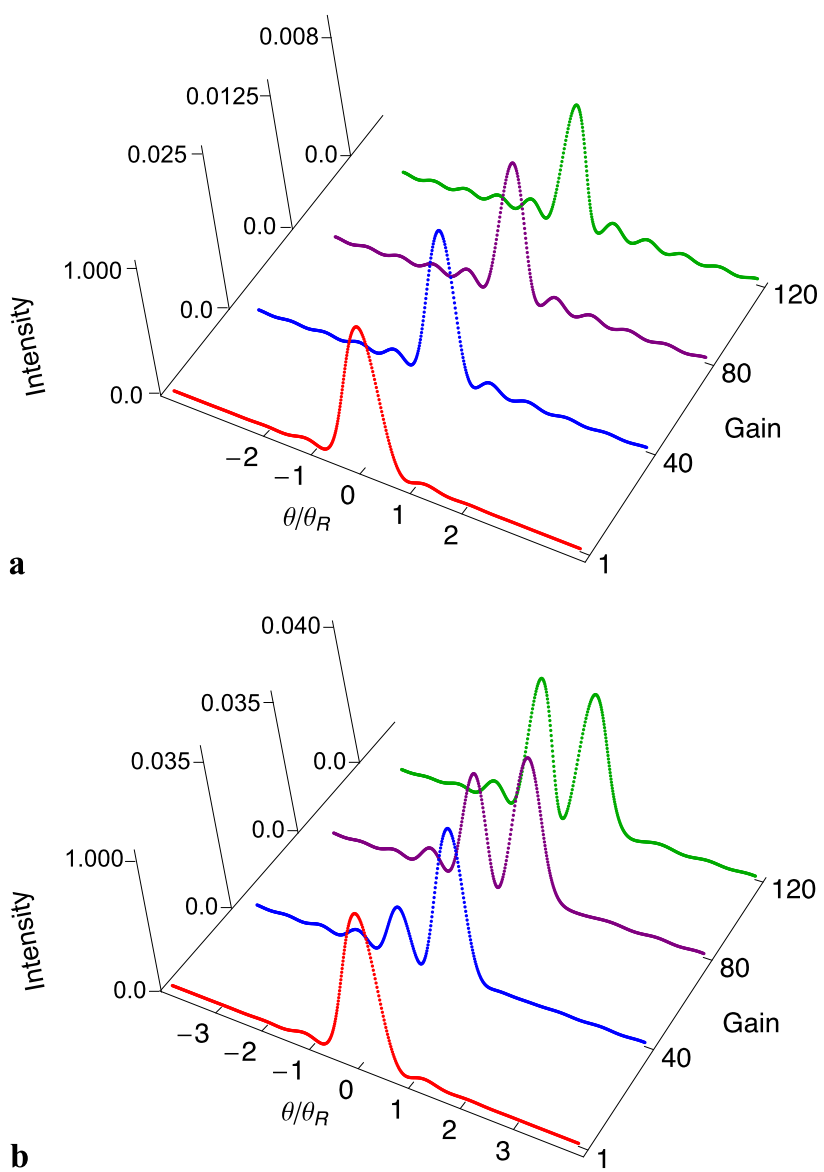

Fig. 4 Intensity distributions in spatial frequency (angular) space for a single on-axis point source and $\mathbf{b}$ two point sources, one on-axis and one off-axis, without $(G=1)$ and with $(G=40,80,120)$ PSA. In (a), the intensity is decreased, while the main characteristics of the shape of the distribution are preserved. In (b), the separation of the two peaks increases, improving the distinguishability of two points

The correlation between the measured signal and the hypothesis $H_{k}$ can be quantified using the likelihood ratio [1]:

$g=\sum_{i} n_{i k} \ln \left(n_{i 1} / n_{i 0}\right)$.

Here, $n_{i 1}$ and $n_{i 0}$ are the mean numbers of photons at the position $x_{i}$ under hypotheses $H_{1}$ and $H_{0}$, respectively, while $n_{i}$ is the detected number of photons at the position $x_{i}$. The ratio $n_{i 1} / n_{i 0}$ is given by [1]:

$n_{i 1} / n_{i 0}=\frac{M_{1}\left(x_{i}\right)}{M_{0}\left(x_{i}\right)}$

According to the central limit theorem, when the number of incident photons is large, the likelihood ratio $g$ is well described by a Gaussian distribution, with mean value [1]:

$\bar{g}_{k}=\int M_{k}(\mathrm{x}) \ln \left[M_{1}(x) / M_{0}(x)\right] d x$ 

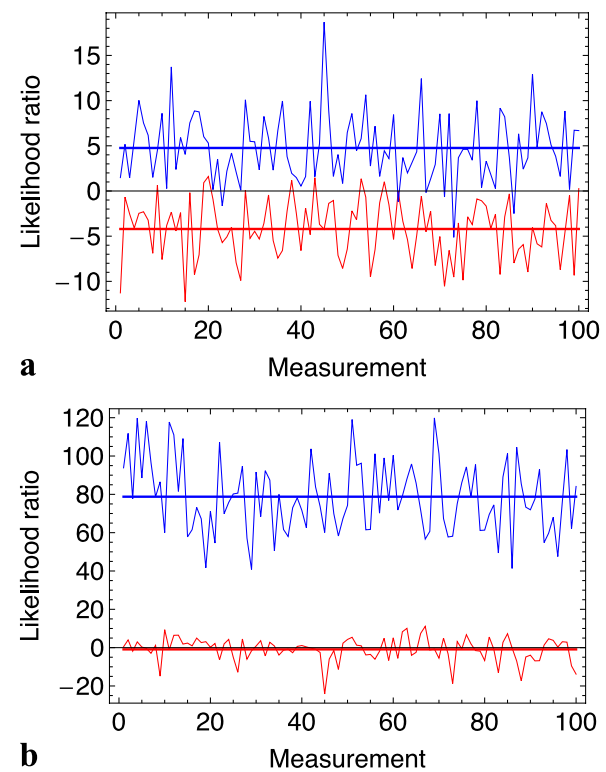

Fig. 5 Likelihood ratios for two hypotheses with a PSA turned off and b PSA turned on in 100 simulated individual measurements. The red and blue curves represent the likelihood ratios for hypothesis 0 and 1 , respectively. The straight lines show the average value of likelihood ratios. In this example, the number of incident signal photons is 100 and the chosen gain of PSA is 10

and variance:

$\sigma_{k}^{2}=\int M_{k}(x) \ln ^{2}\left[M_{1}(x) / M_{0}(x)\right] d x$.

The likelihood ratio calculated for the two hypotheses is compared with a pre-set threshold, $g_{\mathrm{T}}$, to choose the correct hypothesis. The threshold $g_{\mathrm{T}}$ is obtained from the varianceweighted likelihood ratios for two hypotheses [14]:

$g_{\mathrm{T}}=\frac{\sigma_{0} g_{1}+\sigma_{1} g_{0}}{\sigma_{1}+\sigma_{0}}$.

Here, $g_{1}$ and $g_{0}$ represent the likelihood ratios under hypotheses $H_{1}$ and $H_{0}$, respectively, and $\sigma_{1}^{2}$ and $\sigma_{0}^{2}$ are the corresponding variances. An example of the calculation of the likelihood ratio for a fixed number of incident photons exhibiting a Poissonian distribution is shown in Fig. 5. The likelihood ratios shown in Fig. 5 are calculated from the spatial intensity distributions in Fig. 4. Since the chosen number of incident photons is small, the likelihood ratios for the two hypotheses are not well separated before PSA is applied, Fig. 5a. It is thus difficult to identify which hypothesis should be selected. Conversely, the distinction between the likelihood ratios increases when the PSA is introduced into the imaging process, Fig. 5b. In this case, the distinguishability of the two hypotheses is improved after the PSA is applied.

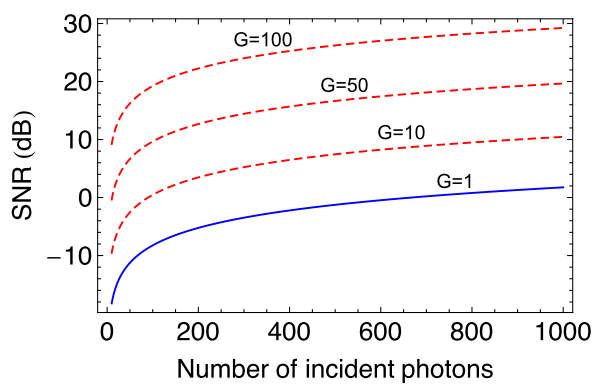

Fig. 6 SNR as a function of the number of incident photons (ranging from 10 to 1000). The SNR improvement is constant when the PSA is used for three example gains: $10,50,100$

Formally, the likelihood SNR for the stated distinguishability problem can be evaluated analytically [1] as

$\mathrm{SNR}=\frac{\left(\bar{g}_{1}-\bar{g}_{0}\right)^{2}}{\sigma_{0}^{2}}=\frac{\left[\int M(x) \ln \left[M_{1}(x) / M_{0}(x) d x\right]^{2}\right.}{\int M_{0}(x) \ln ^{2}\left[M_{1}(x) / M_{0}(x)\right] d x}$,

where $M(x)$ is the difference between expected count-rate densities for two hypotheses:

$M(x)=M_{1}(x)-M_{0}(x)$.

Let $\mathrm{SNR}_{1}$ and $\mathrm{SNR}_{0}$ represent the SNR for PSA turned on and off, respectively. The SNR change, $\triangle \mathrm{SNR}$, associated with PSA can be calculated as:

$$
\begin{aligned}
\Delta \mathrm{SNR}(\mathrm{dB}) & =10 \ln \mathrm{SNR}_{1}-10 \ln \mathrm{SNR}_{0} \\
& =10 \ln \frac{\mathrm{SNR}_{1}}{\mathrm{SNR}_{0}} .
\end{aligned}
$$

As can be seen from (9), the SNR is approximately proportional to the number of incident photons; therefore the change of SNR is invariant with respect to the photon number.

A semi-continuous intensity distribution in spatial frequencies is used for the calculation, corresponding to 100 points over the range shown in Fig. 4. The analytically calculated SNR with the PSA turned off $(G=1)$ and on $(G=1)$ is shown in Fig. 6, as a function of the number of incident photons. SNR increases with the number of incident photons. The rate of increase is greater when the number of incident photons is small, and the SNR improves when the PSA is utilized.

When the signals are exceptionally weak compared to the background, the threshold SNR is used [1], and can be obtained by taking $M(x) \ll M_{0}(x)$, that is [1],

$\mathrm{SNR}=\int \frac{[M(x)]^{2}}{M_{\mathrm{b}}(x)} d x$,

where $M_{\mathrm{b}}(x)$ is the count-rate density for background. In our further calculation, we assume $M_{\mathrm{b}}$ to be uniform and utilize the threshold SNR. 


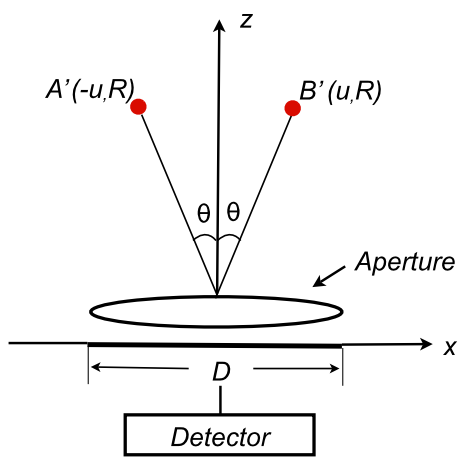

Fig. 7 Schematic of two symmetrically separated point sources, $A^{\prime}$ and $B^{\prime}$, which are located at $(-u, R)$ and $(u, R)$ with a separation angle $2 \theta$

\subsection{Ideal multimode PSA SNR scalings}

The details of the redistribution of spatial frequencies shown in Fig. 4 depend on the PSA gain. For an ideal singlemode PSA, the threshold SNR scales quadratically with PSA gain for small gains [11]. The intensity distributions for the two hypotheses in the spatial domain are approximated as Gaussian functions of various orders, corresponding to apertures of variable sharpness. A configuration for the hypothesis of two point sources is used, Fig. 7, compared with that of a real PSA in Fig. 2. The modification is limited to the symmetric configuration of two sources with respect to the aperture axis, with no overall change in angular separation between sources. This choice is identical to the one used in prior work by Helstrom [1]. It is also assumed that the total power is identical for both hypotheses.

The total illuminance $J(x, R)$ for each point source is the sum of signal illuminance $J_{\mathrm{S}}(x, R)$ and the uniform background illuminance $J_{\mathrm{b}}(x)$,

$$
\begin{aligned}
J(x, u) & =J_{\mathrm{s}}(x, u)+J_{\mathrm{b}}(x) \\
& =A \exp \left[-\left(\frac{x-u}{R}\right)^{\gamma}\right]+J_{\mathrm{b}}(x),
\end{aligned}
$$

where $A$ is the maximum magnitude of the signal illuminance at the center of source plane. The signal illuminance $J_{\mathrm{s}}(x, u)$ is assumed to be a $\gamma$-order Gaussian. The total illuminance for $H_{0}$ is

$J_{0}(x)=2 J(x, 0)$,

and the total illuminance for $H_{1}$ is

$J_{1}(x)=J(x,-u)+J(x, u)$.

When an ideal PSA with a gain $G$ is applied to the hypothesis testing of point sources, the two illuminances become

$J_{0}^{\prime}(x)=\frac{2}{G} J(x, 0)$

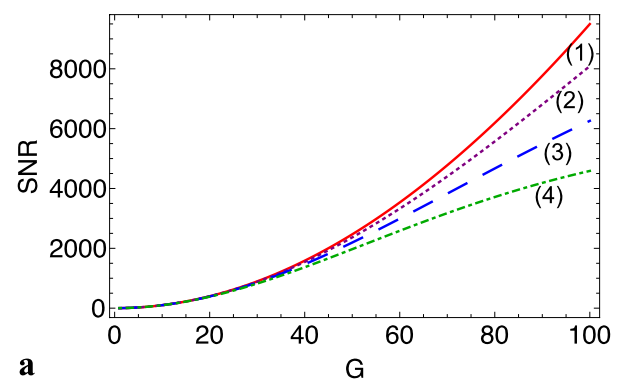

a

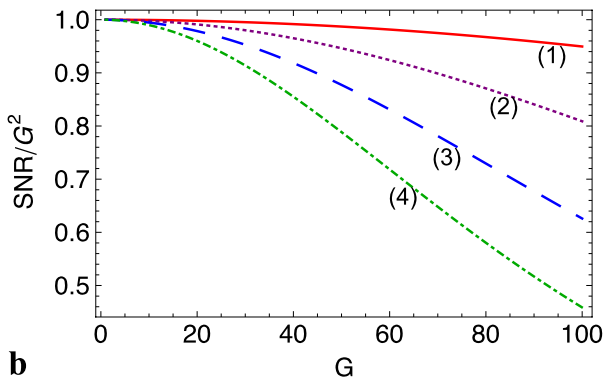

Fig. 8 a Normalized threshold SNR associated with the ideal PSA gain $(G)$ within the range of $1-100$ for several Gaussian orders, $\gamma$ : (1) $\gamma=2$; (2) $\gamma=4$; (3) $\gamma=6$; (4) $\gamma=8$. b Ratio of threshold SNR to $G^{2}$ as a function of gain

and

$J_{1}^{\prime}(x)=\frac{1}{G}[J(x,-G u)+J(x, G u)]$

for $H_{0}$ and $H_{1}$, respectively.

The apparent angle between the two point sources is amplified after utilizing the ideal PSA, which contributes to the improvement of angular resolution. The relationship between the threshold SNR and gain of an ideal PSA is shown in Fig. 8 for Gaussian orders of 2, 4, 6, and 8. It is evident that the quadratic scaling between SNR and gain is only valid for small gains. For higher gains, this scaling is slower than quadratic, and the intensity distributions of higher Gaussian orders result in a slower rate of increase of SNR with gain.

\subsection{Real multimode PSA SNR scalings}

For a real multimode PSA implemented by phase-sensitive optical parametric amplification, we find the performance to be significantly different than that of an ideal multimode PSA, which can be attributed to the differences in the evolution of the spatial frequency spectrum. In an ideal multimode PSA all spatial frequencies experience a constant phase gain and an equal and constant deamplification gain. In contrast, the gain in a real, multimode PSA departs from this idealized condition, as evidenced in Fig. 4. The results of SNR calculations for a realistic multimode PSA are shown in Fig. 9. The increase in SNR for the Gaussian order of 2 is significantly greater than that of greater Gaussian orders. Moreover, when the gain is modified by an offset, the associated 


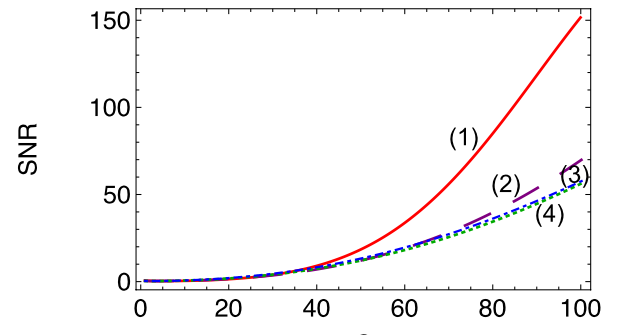

a

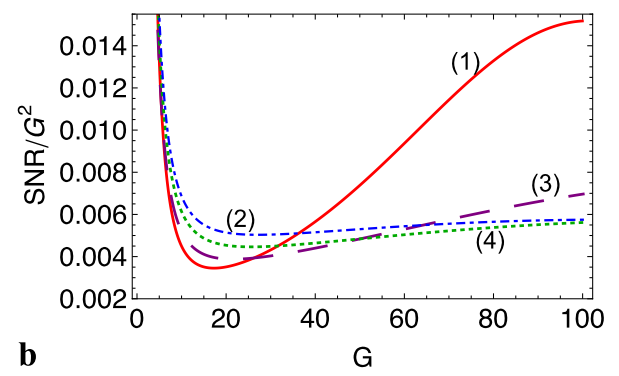

Fig. 9 a Normalized threshold SNR associated with the real PSA gain $(G)$ within the range of $1-100$ for several Gaussian orders $(\gamma)$ : (1) $\gamma=2$; (2) $\gamma=4$; (3) $\gamma=6$; (4) $\gamma=8$. b Ratio of threshold SNR over $G^{2}$ as a function of gain. The initial angular separation in this case is $0.1 \theta_{\mathrm{R}}$

SNR scalings for the Gaussian order of 2 and 6 are greater than quadratic. Thus the SNR performance of a real PSA is different than that of an ideal PSA, and a larger SNR improvement can be achieved by applying the real PSA with high gains. For the Gaussian order of 8 , the scaling of the threshold SNR as a function of gain is studied for different initial separation angles, Fig. 10. A quadratic scaling only applies for higher gains, but there is an offset, i.e. SNR $\propto\left(G-G_{0}\right)^{2}$. The SNR decreases for small gains, which can be understood by studying the evolution of angular distributions in a real PSA, Fig. 4. This effect is attributed to the reduction of the number of photons without angular amplification, while a significant redistribution of spatial frequencies occurs. Another conclusion from this analysis of real PSAs is that a larger initial separation angle, $\theta_{0}$, results in a larger SNR improvement, Fig. 10. Thus the gain that is needed to compensate the SNR loss from the photon number deamplification and recover the SNR will be larger for a smaller $\theta_{0}$.

\section{Conclusions}

It has been demonstrated numerically that the SNR associated with the problem of imaging resolution can be enhanced when a PSA is employed on a transversely varying field distribution. Quadratic scaling of SNR with the PSA gain is deduced from analyzing the threshold SNR. This improvement is restricted by the maximum PSA gain, which theoretically equals to the signal power: $G_{\max }=P_{\mathrm{s}}$,
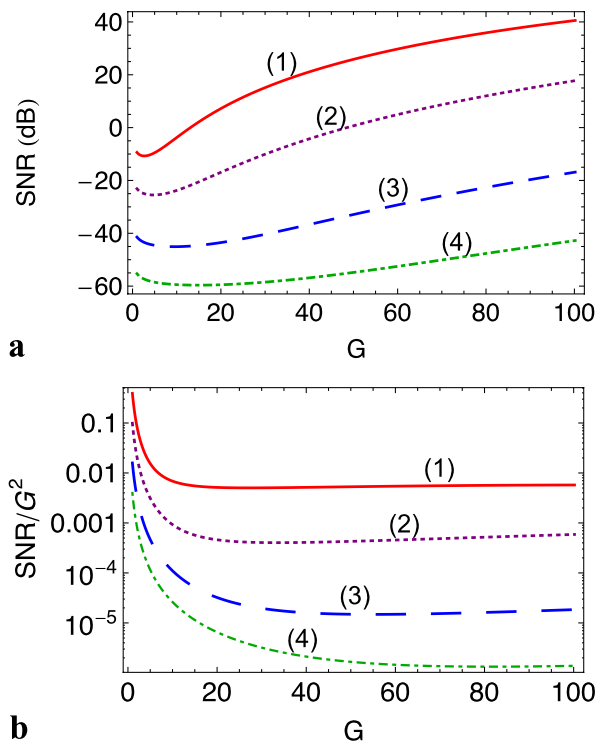

Fig. 10 a Threshold SNR associated with the PSA gain $(G)$ for several different initial angular separations: (1) $\theta_{0} / \theta_{\mathrm{R}}=1 / 10$, (2) $\theta_{0} / \theta_{\mathrm{R}}=1 / 20$, (3) $\theta_{0} / \theta_{\mathrm{R}}=1 / 50$, (4) $\theta_{0} / \theta_{\mathrm{R}}=1 / 100$. b Ratio of threshold SNR and $G^{2}$ as a function of gain. The Gaussian order of the spatial intensity distribution is fixed at 8 in this case

in the units of photon number. Consequently, the maximum resolution improvement in the angular domain that can be achieved for a fixed SNR will be improved by a factor of $1 / P_{\mathrm{s}}$, which shows the angular resolution for point source sensing can approach the quantum limit of the de Broglie resolution (i.e. Heisenberg limit).

PSAs have a potential to enhance the imaging resolution by improving the distinguishability of a single on-axis point source and two asymmetrically distributed point sources. However, different configurations of point source locations can affect the distinguishability improvement of the PSA. Investigation of the application of PSA to distinguishing different configurations of two point sources and resolution of more than two point sources in similar sub-Rayleigh problems is the subject of a future analysis.

Acknowledgements This work has been supported in part by the Defense Advanced Research Projects Agency (DARPA), Contract HR0011-08-1-0066.

Open Access This article is distributed under the terms of the Creative Commons Attribution Noncommercial License which permits any noncommercial use, distribution, and reproduction in any medium, provided the original author(s) and source are credited.

\section{References}

1. C.W. Helstrom, IEEE Trans. Inf. Theory 10, 275 (1964)

2. M.I. Kolobov, Quantum Imaging (Springer, New York, 2007)

3. C.M. Caves, Phys. Rev. D 26, 1817 (1982)

4. M.I. Kolobov, L.A. Lugiato, Phys. Rev. A 52, 4930 (1995) 
5. S.-K. Choi, M. Vasilyev, P. Kumar, Phys. Rev. Lett. 83, 1999 (1938)

6. F. Devaux, J.L. Blanchet, E. Lantz, Opt. Lett. 32, 175 (2007)

7. S. Gigan, L. Lopez, V. Delaubert, N. Treps, C. Fabre, A. Maitre, J. Mod. Opt. 53, 809 (2006)

8. G.M. D'Ariano, C. Macchiavello, N. Sterpi, H.P. Yuen, Phys. Rev. A 54, 4712 (1996)

9. I. Jovanovic, D. French, J. Walter, R. Ratowsky, J. Opt. Soc. Am. B f26, 1169 (2009)
10. R. Bondurant, Opt. Lett. 25, 649 (2000)

11. D. French, Z. Huang, H.-Y. Pao, I. Jovanovic, Phys. Lett. A 373, 999 (2009)

12. L.K. Shalm, R.B.A. Adamson, A.M. Steinberg, Nature (London) 457, 67 (2008)

13. L. Mandel, E. Wolf, Proc. Phys. Soc. 74, 233 (1959)

14. G.P. Agrawal, Fiber-Optic Communication Systems (Wiley, New York, 1997) 